\title{
Intrinsic breast tumor subtypes, race, and long-term survival in the Carolina Breast Cancer Study
}

\author{
Katie M. O'Brien 1 , Stephen R. Cole ${ }^{1}$, Chiu-Kit Tse ${ }^{1,2}$, Charles M. Perou², Lisa A. Carey², \\ William D. Foulkes ${ }^{3}$, Lynn G. Dressler ${ }^{4}$, Joseph Geradts ${ }^{5}$, and Robert C. Millikan ${ }^{1,2}$ \\ ${ }^{1}$ Department of Epidemiology, University of North Carolina, Gillings School of Global Public \\ Health, CB\#7435, University of North Carolina, Chapel Hill, NC 27599, USA \\ ${ }^{2}$ Lineberger Comprehensive Cancer Center, University of North Carolina at Chapel Hill, North \\ Carolina, USA \\ ${ }^{3}$ Program in Cancer Genetics, Departments of Oncology and Human Genetics, McGill University \\ Montreal, QC Canada, H2W 1 S6 \\ ${ }^{4}$ Eshelman School of Pharmacy, Institute of Pharmacogenomics and Individualized Therapy, \\ University of North Carolina at Chapel Hill, North Carolina, USA \\ ${ }^{5}$ Department of Pathology, Duke University Medical Center, Durham NC, USA
}

\begin{abstract}
Purpose-Previous research identified differences in breast cancer-specific mortality across four "intrinsic" tumor subtypes: luminal A, luminal B, basal-like, and human epidermal growth factor receptor 2 positive/estrogen receptor negative (HER2+/ER-).
\end{abstract}

Experimental Design-We used immunohistochemical markers to subtype 1149 invasive breast cancer patients (518 African American, 631 white) in the Carolina Breast Cancer Study, a population-based study of women diagnosed with breast cancer. Vital status was determined through 2006 using the National Death Index, with median follow-up of 9 years.

Results-Cancer subtypes luminal A, luminal B, basal-like and HER2+/ER- were distributed as $64 \%, 11 \%, 11 \%$ and $5 \%$ for whites, and $48 \%, 8 \%, 22 \%$ and $7 \%$ for African Americans, respectively. Breast cancer mortality was higher for patients with HER2+/ER- and basal-like breast cancer compared to luminal A and B. African Americans had higher breast-cancer specific mortality than whites, but the effect of race was statistically significant only among women with luminal A breast cancer. However, when compared to the luminal A subtype within racial categories, mortality for patients with basal-like breast cancer was higher among whites (HR=2.0, 95\% CI: 1.2, 3.4) than African Americans ( $\mathrm{HR}=1.5,95 \%$ CI: 1.0, 2.4), with the strongest effect seen in postmenopausal white women (HR=3.9, 95\% CI: 1.5, 10.0).

Conclusions-Our results confirm the association of basal-like breast cancer with poor prognosis, and suggest that basal-like breast cancer is not an inherently more aggressive disease in African American women compared to whites. Additional analyses are needed in populations with known treatment profiles to understand the role of tumor subtypes and race in breast cancer mortality, and in particular our finding that among women with luminal A breast cancer, African Americans have higher mortality than whites.

\section{Keywords}

Breast Cancer; Breast Cancer Subtypes; Race; Survival; Epidemiology 


\section{Statement of Translational Relevance}

Previous research identified differences in breast cancer-specific mortality across four "intrinsic" tumor subtypes: luminal A, luminal B, basal-like, and human epidermal growth factor receptor 2 positive/estrogen receptor negative (HER2+/ER-). Using data from a population-based study, we observed that African American women had higher breast cancer mortality than whites, but the effect of race was statistically significant only for women with Luminal A breast cancer, a subtype with defined therapeutic targets. Race-stratified estimates for the effect of "intrinsic" subtype on mortality indicated that basal-like breast cancer was not inherently more aggressive in African American women. For smaller tumors, patients with basal-like breast cancer showed greater lymph node involvement than luminal A, while the reverse was true for larger tumors. Heterogeneity was observed in the relationship between tumor subtypes and long-term survival, with cross-over effects after 5 years of follow-up.

\section{Introduction}

Although breast cancer survival has increased substantially over the last 30 years, a large racial disparity remains, with African Americans experiencing higher mortality and shorter survival time than whites [1]. The difference is particularly pronounced among women diagnosed prior to 50 years of age. Prognostic differences between breast tumor subtypes could contribute to the survival disparity, as the subtypes are not equally distributed between race and age groups [2,3]. Breast tumors may be classified using five immunohistochemical (IHC) tumor markers: estrogen receptor (ER), progesterone receptor (PR), human epidermal growth factor receptor-2 (HER2), human epidermal growth factor receptor-1 (HER1) and cytokeratin 5/6 (CK 5/6) [2,4-8].

As demonstrated in a previous analysis of patients from the Carolina Breast Cancer Study (CBCS), although luminal A (ER+ or PR+ and HER2-) is the most common IHC subtype overall, pre-menopausal African American women have a high prevalence of basal-like breast cancer (ER-, PR-, HER2- and either HER1+ or CK 5/6+) [2]. There are currently no targeted therapies for the basal-like subtype, which has higher mortality than the most common subtype, luminal A. Patients with luminal A tumors can be treated with estrogen receptor inhibitors such as tamoxifen or aromatase inhibitors. Tumors expressing HER2 but not ER or PR (HER2+/ER - subtype) were associated with the worst survival in the CBCS [2], although the development of trastuzamab and other HER2-targeted agents to treat HER2+ tumors has since improved prognosis. Carey et al [2] found that the HER2+/ERsubtype was fairly rare in all age and race groups, comprising less than $10 \%$ of cancers in each subpopulation.

Other studies have replicated the finding that basal-like breast cancer is more common in young African Americans [3,9,10] and younger women in Africa [11,12] compared to European American, European, and Asian women [3-8,13-21]. Additionally, nearly all studies agree that basal-like and HER2+/ER- negative tumors have poorer prognoses than luminal A, regardless of the source population [3,4,6,7,10,15-24]. However, many of these studies used only three IHC markers (ER, PR, and HER2) to classify subtypes, thus combining all basal-like and unclassified cancers into a single 'triple negative' subtype. As several studies have corroborated that these two subtypes are biologically and prognostically distinct $[4,7,13,15,22-23]$, subtype misclassification could substantially bias effect estimates. 
Despite the abundance of studies on this topic, only the original CBCS study [2], an Atlantabased case-control study [3], and a California-based cancer registry study [20] have compared IHC subtype frequency distributions in multiracial, population-based samples. Of those, only one study presented racial differences in survival by IHC subtype [3], albeit with ER, PR and HER 2 markers only, and none have examined survival differences by race and menopausal status. For this reason, an updated analysis of the CBCS data, which includes an additional 4 years of follow-up and nearly 1000 more patients enrolled during Phase II of the study, was conducted to help elucidate the relationship between race, menopausal status, breast cancer IHC subtype and survival. Recognizing that the effect of tumor markers may vary over time [25,26], we conducted these analyses using flexible models in addition to standard statistical techniques.

\section{Methods}

\section{Study Population}

The CBCS is a population-based, case-control study conducted in 24 counties of North Carolina (NC). Invasive breast cancer patients diagnosed from 1993-1996 (Phase I) and 1996-2001 (Phase II) were identified using rapid case ascertainment in cooperation with the NC Central Cancer Registry. Survival results were previously published for Phase I [2]. For both phases of the CBCS, patients were selected using weighted sampling probabilities for each race and age subgroup to ensure approximately equal numbers of pre- and postmenopausal African Americans, and pre- and post- menopausal whites [27]. Overall, 1808 women with breast cancer were enrolled, 1149 of which had tumor tissue available for subtype analysis. This included 238 pre-menopausal African Americans, 280 postmenopausal African Americans, 323 pre-menopausal whites and 308 postmenopausal whites.

Race was determined by self-report, with all individuals categorized as either African American or white. Less than $2 \%$ of participants self-identified as multiracial, Hispanic or other race/ethnicities and were classified as white for statistical analyses. Information on menopausal status and other potential covariates were collected during in-home interviews [9]. Women younger than 50 years were considered postmenopausal if they had undergone natural menopause, bilateral oophorectomy, or irradiation to the ovaries, while women older than 50 years were assigned a menopausal status based on menstrual cessation [2]. Tumor size, axillary lymph node status, and stage at diagnosis were abstracted from medical records. ER and PR status were also abstracted from the medical record for approximately $80 \%$ of patients [2].

\section{IHC Subtypes}

The collected tumor tissue was sectioned and stained for a panel of immunohistochemical (IHC) markers at the Immunohistochemistry Core Laboratory at the University of North Carolina, Chapel Hill. Using medical records and markers modeling gene expression profiles of HER2, HER1, CK 5/6, and, if necessary, ER and PR, IHC subtypes were assigned as follows: luminal A (ER+ and/or PR+, HER2-), luminal B (ER+ and/or PR+, HER2+), HER2+/ER- (ER-, PR-, HER2+), basal-like (ER-, PR-, HER2-, HER1+ and/or CK 5/6+) or unclassified (negative for all 5 markers). A more detailed description of the development of these IHC markers as proxies for gene expression analysis can be found elsewhere [2,28,29].

\section{Outcome Assessment}

Participants were matched to National Death Index (NDI) recorded deaths occurring prior to January 1,2007. True matches were determined using weighted probabilistic scores and $a$ 
priori matching cut-points to establish a maximum of one match per individual. The NDI also provided date of death and cause of death for each expired individual. The sensitivity of National Death Index search is estimated to $98 \%$ and specificity approximately $100 \%$ [30]. Using International Classification of Disease (ICD) codes, we categorized cause of death as either breast cancer-specific (ICD-9 174.9 or ICD-10 50.9) or other cause of death based on the first listed primary cause of death.

\section{Statistical Analysis}

We first performed descriptive analyses of age, menopausal status, stage, IHC subtype, hormone receptor status, vital status and cause of death for each racial group. Frequency distributions were adjusted for the sampling probabilities used to identify the appropriate proportion of eligible patients in each race and age group (Phase I: 100\% of African Americans $<50,75 \%$ of African Americans $\geq 50,67 \%$ of whites $<50$, and $20 \%$ of whites $\geq 50$; Phase II: $100 \%$ of African Americans, $50 \%$ of whites $<50$, and $20 \%$ of whites $\geq 50$ ).

After censoring living individuals at December 31, 2006, we modeled breast cancer-specific and overall survival curves by race, menopausal status, IHC subtypes, and ER, PR, and HER2 status using the Kaplan-Meier method. For the breast cancer-specific analysis, we censored individuals who died of causes other than breast cancer at time of death. We conducted additional analyses combining the luminal A and B subtypes and excluding unclassified individuals. Survival curves were compared using a log-rank test, and log cumulative hazards plots were examined for possible deviation from proportional hazards assumptions.

We then conducted survival comparisons for race, menopausal status, IHC subtype, and hormone receptor status using Cox proportional hazards models, regardless of whether proportional hazards assumptions were met. We selected age, race, and date of diagnosis as covariates with the aid of a directed acyclic graph [31,32], a technique that uses a priori knowledge of the relationship between the main exposure, possible covariates, and survival to determine the set of necessary adjustment variables. Hazard ratios (HRs) were adjusted for age and race because of their known associations with both IHC subtype [2,9] and survival. Date of diagnosis was included in the models as a continuous variable to adjust for secular changes in breast cancer diagnosis, assessment, and treatment over the enrollment period.

As there is evidence that IHC subtype can be assessed in precancerous in situ lesions [9], stage at diagnosis could represent an intervening variable between IHC subtype and breast cancer mortality. Therefore, adjusting for stage at diagnosis could potentially bias HR estimates [33]. However, stage at diagnosis also serves as a proxy for treatment, and analyses of breast cancer survival commonly adjust for stage. Thus, we present models adjusted and not adjusted for stage at diagnosis in addition to age, race and date of diagnosis. We excluded a number of potential confounding variables, such as socioeconomic status (income, education), and body size (body mass index, waist hip ratio) because we only had information on baseline measures, and we felt that adjusting for non-time varying estimates would be insufficient and potentially biasing if they were affected by a woman's breast cancer diagnosis. No treatment information was available for participants in the CBCS.

After conducting these survival analyses in the entire study population using both adjustment sets (i.e. race, age, and date of diagnosis or race, age, date of diagnosis and stage of disease), we then completed the same analyses within racial strata using both adjustment sets (excluding race), and within strata defined by race and menopausal status, adjusting for age and enrollment year only. We also estimated hazard ratios (HRs) for the effect of stage, 
tumor size, lymph node status or presence of metastatic disease on mortality within each subtype strata to better evaluate the interaction between stage and IHC subtype. These models were adjusted for race, age and date of diagnosis. Additionally, the relationship of axillary lymph node status (percent of patients exhibiting lymph nodes positive for malignancy, average number of positive nodes, and percent of positive nodes for each patient) and tumor size $(\leq 2 \mathrm{~cm}, 2-5 \mathrm{~cm},>5 \mathrm{~cm})$ was examined using Chi-square and Wilcoxon rank sum tests.

To assess departure from proportional hazards assumptions, we examined 1-, 2-, or 3degree polynomial time by exposure interaction terms. We also modeled the change in the HR over time using restricted cubic splines [34], with knots at the $5^{\text {th }}, 35^{\text {th }}, 65^{\text {th }}$, and $95^{\text {th }}$ percentiles. We examined the polynomial and spline models for patterns in how the HRs changed over time to identify meaningful cut-points for appropriate time-stratified proportional hazards models. All analyses were conducted using SAS version 9.2 (SAS Institute, Cary NC) and all figures were created using R 2.9.0 (The R Foundation for Statistical Computing, http://www.r-project.org/foundation).

\section{Results}

\section{Population characteristics}

Characteristics of study participants are presented in Supplementary Table 1. African American patients were younger than white patients, more likely to be pre-menopausal ( $41 \%$ versus $32 \%$ ), diagnosed at later stage (65\% versus $48 \%$ at Stage II or higher) and have ERnegative (51\% versus $32 \%$ ) or PR-negative (55\% versus $36 \%$ ) disease. The proportion of patients with HER2-positive disease was similar in African Americans and whites (15\% and $18 \%$, respectively). The distribution of "intrinsic" IHC subtypes according to race and menopausal status indicated that although luminal A was the most common subtype of breast cancer overall $(57 \%, 67 \%, 40 \%$ and $55 \%$ of premenopausal white, postmenopausal white, premenopausal African American and postmenopausal African American women, respectively), premenopausal African American women exhibited a higher percentage of basal-like tumors (29\%) compared with premenopausal whites (15\%), postmenopausal whites (10\%), and postmenopausal African Americans (17\%).

\section{Survival statistics}

Median follow-up time was 9.0 years (range 0.2 to 13.7 years). There were 347 total deaths, with 239 due to breast cancer. Time plots for a 5\% random sample of participants can be seen in the supplement (Supplementary Figures 1 and 2). We estimated the five-year risk of death due to any cause as $17 \%$ and the five-year risk of death due to breast cancer as $14 \%$ (Kaplan-Meier curve and table found in Supplementary Figure 3). African American women were more likely to die of breast cancer than whites, with 17\% 5-year breast cancer-specific mortality, versus $11 \%$ for whites (Supplementary Table 2). Patients with luminal A tumors had the lowest 5-year breast cancer-specific mortality (9\%), followed by luminal B (12\%). Women with HER2+/ER- tumors exhibited the highest breast cancer-specific mortality, with $26 \%$ deaths within 5 years of diagnosis, followed by basal-like and unclassified, with $24 \%$ and $18 \%$ deaths, respectively.

Survival statistics and HRs for breast cancer-specific mortality for all study participants combined are presented in Supplementary Table 3. Statistically significant differences in breast cancer-specific mortality were observed according to race, menopausal status, IHC subtype, ER, PR, and HER2 status (P-value for log rank test less than 0.05). African Americans had higher breast-cancer specific mortality than whites (HR $=1.7,95 \%$ CI; 1.3 , 2.2), even after adjustment for stage at diagnosis (HR=1.7, 95\% CI: 1.3, 2.2), or stage and 
IHC subtype (HR=1.6, 95\% CI: 1.2, 2.0). Analyzed as single markers, patients with HER2+ tumors did not have a worse prognosis than patients with ER-negative or PR-negative tumors $(\mathrm{HR}=1.5,95 \% \mathrm{CI}: 1.1,2.0 ; \mathrm{HR}=1.6,95 \% \mathrm{CI}: 1.2,2.1$; and $\mathrm{HR}=1.7,95 \% \mathrm{CI}: 1.3$, 2.2 for the effect of HER2, ER, and PR, respectively), particularly after adjusting for stage at diagnosis ( $\mathrm{HR}=1.2,95 \%$ CI: $0.9,1.7 ; \mathrm{HR}=1.4,95 \%$ CI: $1.1,1.8$; and $\mathrm{HR}=1.5,95 \%$ CI: $1.1,1.9)$. However, when these tumor markers were considered jointly and incorporated into the five-marker scheme defining "intrinsic" IHC subtypes, a different pattern emerged. Among all patients, women with HER2+/ER - disease had the highest risk of death (HR= 2.3, 95\% CI: 1.5, 3.6), followed by basal-like (HR=1.7, 95\% CI: 1.2, 2.4). Survival statistics and HRs were also estimated for overall (all-cause) mortality (data not shown). HRs were more precise but slightly attenuated using overall (all-cause) mortality as the outcome (data not shown).

\section{Subtype and race}

Race-stratified Kaplan-Meier plots and HRs for the effect of race (African American versus white) for each IHC subtype are presented in Figure 1. Although African American patients showed higher breast cancer-specific mortality than whites for each IHC subtype, the effect of race was statistically significant only among women with Luminal A breast cancer. Adjustment for stage at diagnosis did not substantially change the magnitude of the HRs, with the exception of the HER2+/ER- subtype, which showed a stronger association with race after adjustment for stage.

Race-stratified survival results are presented in Table 1. HRs for menopausal status, ER, PR, and HER 2 were fairly similar for African Americans and whites, as were most of the racestratified IHC subtype effect estimates. HRs for basal-like breast cancer compared to Luminal A were slightly higher among whites than African Americans. Adjustment for stage at diagnosis resulted in HRs of similar direction and magnitude for race and menopausal status, but resulted in attenuated effect estimates for IHC subtype and hormone receptor status. The most extreme example was among whites, where the HR for HER2+/ER-versus luminal A breast cancer was 2.4 when adjusted for age, and date of diagnosis, but only 1.4 when adjusted for age, date of diagnosis, and stage.

\section{Subtype, race and menopausal status}

HRs stratified according to race and menopausal status are presented in Table 2. HRs were imprecise owing to small sample size within strata. For the IHC subtype analysis, HRs were highest among postmenopausal whites with either HER2+/ER- or basal-like breast cancer, compared to luminal A. The HER2+/ER - subtype consistently had the highest HR for each patient subgroup. Importantly, HRs for basal-like breast cancer were slightly higher among premenopausal white compared to premenopausal African American patients, and higher among postmenopausal white compared to postmenopausal African American patients. HRs for PR- vs. PR+, and HER2+ vs. HER2- were highest for postmenopausal whites, while the HR for ER- vs. ER+ was highest among postmenopausal African American patients. When these models were additionally adjusted for stage at diagnosis, effect estimates were similar but attenuated.

\section{Flexible modeling techniques}

Results from polynomial time-interaction models indicated that the proportional hazards assumption was valid when comparing breast cancer-specific mortality by menopausal status $(\mathrm{P}=0.8)$ or HER status $(\mathrm{P}=0.4)$, but the assumption was violated when comparing race, ER status, PR status, IHC subtype, and combined IHC subtype ( $\mathrm{P} \leq 0.01$ for each test) (data not shown). For ER status, PR status and IHC subtype, a one-degree polynomial time- 
interaction provided the best model fit, but race and combined subtype required cubic timeinteraction terms.

After determining the best fit model for each exposure, we plotted the data to investigate how the HR changed over time for each analysis. For example, the best polynomial model for the subtype analysis was a 1-degree time interaction model, so this appears as 4 diagonal lines, one for each subtype comparison (versus luminal A). Spline models were also plotted for each exposure. Plots showing the best-fitting polynomial and spline models for breast cancer-specific mortality and subtype are presented in Supplementary Figure 4. Based on these plots, we concluded that stratifying HR estimates into two time periods, $0-5$ years and greater than 5 years, would be an appropriate way to capture heterogeneity in the HRs over time without sacrificing interpretability of the effect estimates. Five years of follow-up was the point where most HRs approached the null or changed directions for both the polynomial and spline models.

Time-stratified estimates for breast cancer-specific mortality are provided in Table 3. Single estimates are provided for models that did not violate the proportional hazards assumptions (menopausal status, HER2 status). Effect estimates for basal-like and unclassified breast cancer were greater than 1 in the first 5 years and less than 1 after 5 years of follow-up. The HR for HER2+/ER- was higher in the first five years but remained greater than 1, while the HR for luminal B increased slightly with time. HRs for ER and PR were greater than 1 during the first 5 years, but dropped to 1.0 or less than 1.0 for the second time period. HRs for African American race were greater than 1 and statistically significant for both time periods. HRs adjusted for stage showed similar patterns but were closer to the null (data not shown).

\section{Subtypes and stage}

HRs for stage at diagnosis, tumor size, number of positive axillary lymph nodes and presence of metastatic disease, overall and stratified by IHC subtype, are presented in Table 4. For all subtypes, later stage at diagnosis, larger tumor size, increasing number of axillary lymph nodes, and presence of metastatic disease resulted in higher mortality. Trends were increasing and monotonic, but differed slightly between subtypes. For basal-like tumors, the HRs for a tumor measuring $2-5 \mathrm{~cm}$ versus $<2 \mathrm{~cm}$ was 1.5 (95\% CI: $0.8-2.9$ ), whereas for luminal A, luminal B and HER2+/ER- the HRs were greater than 2.0. For larger tumors $(>5$ $\mathrm{cm}$ ), basal-like and luminal B tumors exhibited lower HRs than luminal A and HER2+/ER-. It should be noted that CIs were wide and overlapped across IHC subtypes.

\section{Subtypes and lymph node status}

To further explore the relationship between IHC subtypes, tumor size and variables related to axillary lymph node status, additional analyses were conducted. As presented in Table 5, a stronger association was observed for each lymph node variable and increasing tumor size for luminal A, luminal B and HER2+/ER-, unclassified, but not basal-like tumors.

Additional analyses were conducted comparing the distribution of lymph node variables in basal-like versus luminal A patients. For tumors $\leq 2 \mathrm{~cm}$, patients with basal-like breast cancer had a higher percentage of lymph node positive tumors $(\mathrm{P}=0.04)$, more positive lymph nodes per patient $(\mathrm{P}=0.03)$, and a higher percentage of positive lymph nodes per patient $(\mathrm{P}=0.01)$ than women with luminal A breast cancer. However, in larger tumors, the reverse was true. For tumors $>2-5 \mathrm{~cm}$, each of the lymph node variables was higher among luminal A compared to basal-like patients ( $\mathrm{P}=0.02$ for all 3 tests of association). 


\section{Discussion}

The prognostic significance of "intrinsic" IHC breast cancer subtypes was determined using data from the Carolina Breast Cancer Study, a population-based epidemiologic study of African American and white women in North Carolina with a median follow-up time of 9 years. We evaluated how hazard ratios for IHC subtypes were modified by race, menopausal status, and duration of follow-up, and also examined the contributions of tumor size, lymph node status and presence of metastatic disease. HRs for race, IHC subtypes, ER, PR, HER2 status and stage at diagnosis were consistent with previously reported data from other recent studies $[3,4,6,7,10,13,15-26]$. Patients with HER2+/ER- breast cancer had the worst prognosis, followed by basal-like. The CBCS was conducted prior to the introduction of trastuzumab and other HER2-targeted agents to treat HER2+ tumors.

Race-stratified analyses in the CBCS indicated that breast cancer mortality was higher for African American women compared to white women, even after adjustment for stage at diagnosis and "intrinsic" IHC subtype. However, the effect of race was statistically significant only among women with Luminal A breast cancer. HRs for basal-like breast cancer compared to luminal A were slightly higher in white patients compared to African Americans, and this was true among pre- as well as postmenopausal women. Thus, basallike breast cancer does not appear to be an inherently more aggressive in African American women compared to whites in the CBCS. Racial differences in breast cancer mortality are likely to be driven by differences in treatment and access to care (as represented by income or education) for Luminal A and other subtypes of breast cancer [35], in addition to the higher prevalence of basal-like breast cancer among younger African American women.

Although CBCS results generally agree with those reported by Lund et al [3], our analyses provide more precise estimates with additional information about the relative prognostic importance of each IHC subtype within racial strata and we were able to provide separate estimates for basal-like and unclassified tumors. Classical prognostic factors such as stage at diagnosis, tumor size, number of affected lymph nodes and presence of metastases were predictive of mortality across all IHC subtypes. As reported in previous studies $[14,36]$, we observed a somewhat attenuated relationship between tumor size and survival for basal-like breast cancer, particularly when comparing HRs across IHC subtypes for tumors $>2-5 \mathrm{~cm}$ in size. For tumors of $>2-5 \mathrm{~cm}$, only $41 \%$ of basal-like breast tumors exhibited positive axillary lymph nodes, a lower percentage than for other IHC subtypes (range $47 \%$ to $61 \%$ ). Lower levels of lymph node metastasis could possibly contribute to the superior survival seen for patients with larger basal-like breast tumors compared with patients diagnosed with similarly sized tumors of other subtypes. Conversely, higher levels of lymph node metastasis in smaller basal-like tumors could contribute to poor prognosis in this group of patients. Since stage at diagnosis was predictive of mortality for each IHC subtype, and subtype was predictive of mortality with and without adjustment for stage, we deduce that both IHC subtype and stage affect prognosis and need to be evaluated together in the clinical setting.

One debatable issue is whether breast cancer-specific or all-cause mortality should be the main outcome of interest. We chose to focus on breast cancer-specific mortality as the main outcome, because we were interested in evaluating the effects of specific breast tumorrelated markers on survival time. However, since misclassification could have occurred if a death was breast-cancer related, but not primarily due to breast cancer (e.g. heart disease related to chemotherapy), or if the physician filling out the death certificate misattributed cause of death (e.g. stating that death was due to lung cancer instead of breast cancer metastasized to the lung), we conducted analyses using both outcomes. If most of the causes of death were correctly attributed, we would expect to observe weaker but more precise estimates for all-cause mortality than for breast cancer-specific mortality. Because this 
pattern was observed in our data, we conclude that valid inferences can be drawn based upon breast cancer-specific mortality.

Statistical analyses using flexible modeling techniques for time-to-event data demonstrated that HRs for IHC subtypes varied over time. Interestingly, for basal-like and unclassified tumors, the time-stratified effect estimates were on the opposite sides of the null. This suggests that individuals who were triple negative for ER, PR and HER2 (which includes basal-like, claudin-low and unclassified tumors) had higher mortality initially, when compared to patients with luminal A breast cancer, but lower mortality once they survived the first 5 years. Similar findings were reported by Dent et al [36,37] and Tischowitz et al. [38]. With respect to breast cancer recurrence, several studies have shown high risk of early relapse among hormone receptor-negative and triple-negative breast cancer patients, and a more constant rate of relapse for hormone-receptor positive disease [39]. Some of the crossover effect may be due to the fact that only surviving women in each stratum are included when estimating effects for later time periods [40].

Over twenty years ago, Gore et al. [41] reported non-proportional hazards for breast in relation to menopausal status, nodal status, tumor size and other clinical variables, and recommended a step-wise analysis of breast cancer survival in 5-year increments. While the 5 -year cut-point is somewhat arbitrary, statistical tests of proportional hazards assumptions using CBCS data indicated deviations from a constant HR. We used graphs of the survival functions, polynomial models and spline models to select 5 years of follow-up as the optimal cut-point for time stratification. Choosing defined cut-points for time-stratification allows for a much simpler interpretation of hazard functions than would be possible with a polynomial or spline model, but still allows for heterogeneity in HRs over time [25,26,34]. Regardless of the exact stratification point, we feel strongly that time-to-event analyses should carefully evaluate proportional hazards assumptions and be open to exploring more flexible models, as such methods may reveal information about the nature of exposureoutcome relationship that cannot be captured using standard techniques. The fact that HRs for IHC subtypes vary over time could have important policy implications in terms of monitoring breast cancer survivors.

Our study has several limitations. The differences we observe by subtype and race may be due to unobserved treatment differences by race. In addition, there is the potential for misclassification of one or more covariates. Since the IHC tumor markers are proxies for gene expression profiling, there may have been some misclassification of intrinsic breast cancer subtype. Further misclassification may have occurred if medical record reports of ER and PR status or disease stage were inaccurate, although previous exploration of this issue among CBCS patients revealed that IHC cut-offs for receptor positivity were reasonably standardized across all included laboratories [42,43]. Although some causes of death may have been misattributed, validation studies showed the NDI to be highly accurate $[44,45]$, indicating that misclassification should not have substantially biased our findings.

Although all participants had complete data for race, age, and date of diagnosis, several had missing data for stage at diagnosis, and 659 otherwise eligible individuals were excluded entirely because of missing IHC subtype information. Analyses comparing patients with and without IHC subtype information showed that the two groups were very similar in terms of age, menopausal status, ER and PR status, and vital status, but those with missing subtype information were more likely to have HER2-negative disease, lower stage at diagnosis, smaller tumors, fewer positive lymph nodes, and were less likely to have died from their breast cancer. Because stage at diagnosis and HER status, in particular, have strong effects on mortality, excluding these individuals may have biased our results. However, this bias is likely towards the null since patients with HER2-negative and/or lower stage disease are 
more likely to have luminal A disease, thus removing patients with longer survival times from the referent group for HR estimation. Thus, differences in survival between patients with luminal A compared to the other IHC subtypes may be even greater than observed in our study.

Limited follow-up information in the CBCS makes it impossible to examine racial disparities in access to care and associated mortality differences or to perform analyses using the shorter endpoint of breast cancer recurrence. Although this was by far the largest multiracial study of breast cancer IHC subtypes and survival time, the sample size was still not large enough to produce precise effect estimates in subgroups defined by menopausal status and race. Therefore, we believe that additional analyses are needed in other equally diverse populations with known treatment profiles to more fully understand the role of intrinsic subtypes and race in breast cancer mortality.

Our study had several strengths. The study population was a large, population-based sample, with sampling probabilities used to obtain unique diversity by race and age at diagnosis. The population-based design facilitated ascertainment of a full spectrum of disease stages, which is often not available in clinical trial-based samples. Another strength was that each participant was given an extensive home interview and asked to provide medical records and tumor samples. This provided us with detailed information on relevant covariates and allowed us to conduct IHC analyses to assess cancer subtypes. Finally, because participant follow-up began just after diagnosis and continued for an extended period of time using a reliable data resource, there was minimal loss to follow-up.

In conclusion, long-term survival analysis of CBCS patients confirms the association of basal-like breast cancer with poor prognosis. While our data show a higher prevalence of basal-like breast cancer in younger African American patients, basal-like breast cancer does not appear to be an inherently more aggressive disease in African American women compared to whites. Disparities in access to care, including established treatments for ER+ (luminal A) breast cancer, could also contribute to higher breast cancer mortality in African American women. Additional analyses are needed in populations with known treatment profiles and recurrence data to more fully understand the role of tumor subtypes and race in breast cancer mortality. Flexible statistical models that address heterogeneity for $0-5$ years versus greater than 5 years of follow-up will be needed, as recently demonstrated for ER+ and ER- breast cancer patients in the National Surgical Adjuvant Breast and Bowel Project [46]. We also found that patients with larger basal-like tumors have on average fewer positive lymph nodes and a better prognosis relative to patients with other "intrinsic" subtypes. The complex interplay of tumor size, lymph-node status and prognosis is an important area of investigation [47], and it will be interesting to determine whether our findings are replicated in other patient populations.

\section{Supplementary Material}

Refer to Web version on PubMed Central for supplementary material.

\section{Acknowledgments}

This research was funded in part by the University Cancer Research Fund of North Carolina, the National Cancer Institute Specialized Program of Research Excellence (SPORE) in Breast Cancer (NIH/NCI P50-CA58223), and the Lineberger Comprehensive Cancer Center Core Grant (NIH/NCI P30-CA16086). KO was funded by a training grant from the National Institute of Health (NIH/NCI T32-CA009330). WDF would like to thank the Turner Family Cancer Research Fund for financial support. The authors acknowledge the technical assistance of David Cowan and Debbie Little, Immunohistochemistry Core Facility at UNC. The authors wish to thank the women who participated in the CBCS. The authors have no conflicts of interest to declare.

Clin Cancer Res. Author manuscript; available in PMC 2011 December 15. 
Grants:

This research was funded in part by the University Cancer Research Fund of North Carolina (KMO, CKT, CMP, LAC, LGD, JG and RCM), the National Cancer Institute Specialized Program of Research Excellence (SPORE) in Breast Cancer (NIH/NCI P50-CA58223) (KMO, CKT, CMP, LAC, LGD, JG and RCM), the Lineberger Comprehensive Cancer Center Core Grant (NIH/NCI P30-CA16086) (KMO, CKT, CMP, LAC, LGD, JG and RCM), a training grant from the National Institute of Health (NIH/NCI T32-CA009330) (KMO), and the Turner Family Cancer Research Fund (WDF).

\section{References}

1. Seer.cancer.gov [Internet]. Bethesda: National Cancer Institute; [cited 2010 January 12]. Available from http://seer.cancer.gov

2. Carey LA, Perou CM, Livasy CA, et al. Race, breast cancer subtypes, and survival in the Carolina Breast Cancer Study. JAMA. 2006; 295:2492-2502. [PubMed: 16757721]

3. Lund MJ, Trivers KF, Porter PL, et al. Race and triple negative threats to breast cancer survival: a population-based study in Atlanta, GA. Breast Cancer Res Treat. 2009; 113:357-70. [PubMed: 18324472]

4. Cheang MC, Voduc D, Bajdik C, et al. Basal-like breast cancer defined by five biomarkers has superior prognostic value than triple-negative phenotype. Clin Cancer Res. 2008; 14:1368-76. [PubMed: 18316557]

5. Yang XR, Sherman ME, Rimm DL, et al. Differences in risk factors for breast cancer molecular subtypes in a population-based study. Cancer Epidemiol Biomarkers Prev. 2007; 16:439-43. [PubMed: 17372238]

6. Kim MJ, Ro JY, Ahn SH, Kim HH, Kim SB, Gong G. Clinicopathologic significance of the basallike subtype of breast cancer: a comparison with hormone receptor and Her2/neu-overexpressing phenotypes. Human Pathol. 2006; 37:1217-26. [PubMed: 16938528]

7. Kurebayashi J, Moriya T, Ishida T, et al. The prevalence of intrinsic subtypes and prognosis in breast cancer patients of different races. Breast. 2007; 16:S72-77. [PubMed: 17714947]

8. Lin CH, Liau JY, Lu YS, et al. Molecular subtypes of breast cancer emerging in young women in Taiwan: evidence for more than just westernization as a reason for the disease in Asia. Cancer Epidemiol Biomarkers Prev. 2009; 18:1807-14. [PubMed: 19505913]

9. Millikan RC, Newman B, Tse CK, et al. Epidemiology of basal-like breast cancer. Breast Cancer Res Treat. 2007; 109:123-9. [PubMed: 17578664]

10. Ihemelandu CU, Naab TJ, Mezghebe HM, et al. Treatment and survival outcome for molecular breast cancer subtypes in black women. Ann Surg. 2008; 247:463-9. [PubMed: 18376191]

11. Nalwoga H, Arnes JB, Wabinga H, Akslen LA. Frequency of the basal-like phenotype in African breast cancer. APMIS. 2007; 115:1391-9. [PubMed: 18184410]

12. Huo D, Ikpatt F, Khramtsov A, et al. Population differences in breast cancer: survey in indigenous African women reveals over-representation of triple-negative breast cancer. J Clin Oncol. 2009; 27:4515-21. [PubMed: 19704069]

13. Sihto H, Lundin J, Lehtimäki T, et al. Molecular subtypes of breast cancers detected in mammography screening and outside of screening. Clin Cancer Res. 2008; 14:4103-10. [PubMed: 18593987]

14. Foulkes WD, Grainge MJ, Rakha EA, Green AR, Ellis IO. Tumor size is an unreliable predictor of prognosis in basal-like breast cancers and does not correlate closely with lymph node status. Breast Cancer Res Treat. 2009; 117:199-204. [PubMed: 18600446]

15. Shin BK, Lee Y, Lee JB, et al. Breast carcinomas expressing basal markers have poor clinical outcome regardless of estrogen receptor status. Oncol Rep. 2008; 19:617-25. [PubMed: 18288392]

16. Nakajima H, Fujiwara I, Mizuta N, et al. Prognosis of Japanese breast cancer based on hormone receptor and HER2 expression determined by immunohistochemical staining. World J Surg. 2008; 32:2477-82. [PubMed: 18758851]

17. Spitale A, Mazzola P, Soldini D, Mazzucchelli L, Bordoni A. Breast cancer classification according to immunohistochemical markers: clinicopathologic features and short-term survival 
analysis in a population-based study from the South of Switzerland. Ann Oncol. 2009; 20:628-35. [PubMed: 19074747]

18. Zhao J, Liu H, Wang M, et al. Characteristics and prognosis for molecular breast cancer subtypes in Chinese women. J Surg Oncol. 2009; 100:89-94. [PubMed: 19544363]

19. Onitilo AA, Engel JM, Greenlee RT, Mukesh BN. Breast cancer subtypes based on ER/PR and Her2 expression: comparison of clinicopathologic features and survival. Clin Med Res. 2009; 7:413. [PubMed: 19574486]

20. Parise CA, Bauer KR, Brown MM, Caggiano V. Breast cancer subtypes as defined by the estrogen receptor (ER), progesterone receptor (PR), and the human epidermal growth factor receptor 2 (HER2) among women with invasive breast cancer in California, 1999-2004. Breast J. 2009; 15:593-602. [PubMed: 19764994]

21. Muñoz M, Fernández-Aceñero MJ, Martín S, Schneider J. Prognostic significance of molecular classification of breast invasive ductal carcinoma. Arch of Gynecol and Obstet. 2009; 280:43-8. [PubMed: 19082617]

22. Liu H, Fan Q, Zhang Z, Li X, Yu H, Meng F. Basal-HER2 phenotype shows poorer survival than basal-like phenotype in hormone receptor-negative invasive breast cancers. Hum Pathol. 2008; 39:167-74. [PubMed: 18045647]

23. Yamamoto Y, Ibusuki M, Nakano M, Kawasoe T, Hiki R, Iwase H. Clinical significance of basallike subtype in triple-negative breast cancer. Breast Cancer. 2009; 16:260-7. [PubMed: 19701681]

24. Ibrahim E, Al-Gahmi AM, Zeenelin AA, et al. Basal vs. luminal A breast cancer subtypes: a matched case-control study using estrogen receptor, progesterone receptor and HER-2 as surrogate markers. Med Oncol. 2009; 26:372-8. [PubMed: 19034706]

25. Gray RJ. Flexible methods for analyzing survival data using splines, with applications to breast cancer prognosis. J Am Stat Assoc. 1992; 87:942-51.

26. Natarajan L, Pu M, Parker BA, et al. Time-varying effects of prognostic factors associated with disease-free survival in breast cancer. Am J Epidemiol. 2009; 169:1463-70. [PubMed: 19403844]

27. Newman B, Moorman PG, Millikan RC, et al. The Carolina Breast Cancer Study: integrating population-based epidemiology and molecular biology. Breast Cancer Res Treat. 1995; 35:51-60. [PubMed: 7612904]

28. Perou CM, Sørlie T, Eisen MB, et al. Molecular portraits of human breast tumours. Nature. 2000; 406:747-52. [PubMed: 10963602]

29. Sørlie T, Perou C, Tibshirani R, et al. Gene expression patterns of breast carcinomas distinguish tumor subclasses with clinical implications. Proc Natl Acad Sci USA. 2001; 98:10869-74. [PubMed: 11553815]

30. Rich-Edwards JW, Carsano KA, Stampfer MJ. Test of the National Death Index and Equifax Nationwide Death Search. Am J Epidemiol. 1994; 140:1016-9. [PubMed: 7985649]

31. Pearl J. Causal diagrams for empirical research. Biometrika. 1995; 82:669-710.

32. Greenland S, Pearl J, Robins JM. Causal diagrams for epidemiologic research. Epidemiology. 1999; 10:37-48. [PubMed: 9888278]

33. Cole SR, Hernán MA. Fallibility in estimating direct effects. Int J Epidemiol. 2002; 31:163-5. [PubMed: 11914314]

34. Harrell, F. Regression Modeling Strategies, with Applications to Linear Models, Logistic Regression, and Survival Analysis. 1. New York: Springer; 2001.

35. Komenaka IK, Martinez ME, Pennington RE Jr, et al. Race and ethnicity and breast cancer outcomes in an underinsured population. J Natl Cancer Inst. 2010; 102:1178- 1187. [PubMed: 20574040]

36. Dent R, Hanna WM, Trudeau M, Rawlinson E, Sun P, Narod SA. Time to disease recurrence in basal-type breast cancers: effects of tumor size and lymph node status. Cancer. 2009; 115:491723. [PubMed: 19691094]

37. Dent R, Trudeau M, Pritchard KI, et al. Triple-negative breast cancer: clinical features and patterns of recurrence. Clin Cancer Res. 2007; 13:4429-34. [PubMed: 17671126]

38. Tischkowitz M, Brunet JS, Bégin LR, et al. Use of immunohistochemical markers can refine prognosis in triple negative breast cancer. BMC Cancer. 2007; 7:134. [PubMed: 17650314] 
39. Anderson WF, Jatoi I, Devesa SS. Assessing the impact of screening mammography: Breast cancer incidence and mortality rates in Connecticut (1943-2002). Breast Cancer Res Treat. 2006; 99:33340. [PubMed: 16703451]

40. Hernán M. The hazards of hazard Ratios. Epidemiology. 2010; 21:13-15. [PubMed: 20010207]

41. Gore SM, Pocock SJ, Kerr GR. Regression models and non-proportional hazards in the analysis of breast cancer survival. App Stat. 1984; 33:176-95.

42. Huang WY, Newman B, Millikan RC, Schell MJ, Hulka BS, Moorman PG. Hormone-related factors and risk of breast cancer in relation to estrogen receptor and progesterone receptor status. Am J Epidemiol. 2000; 151:703-14. [PubMed: 10752798]

43. Ma H, Wang Y, Sullivan-Halley J, Weiss L, et al. Breast cancer receptor status: do results from a centralized pathology laboratory agree with SEER registry reports? Cancer Epidemiol Biomarkers Prev. 2009; 18:2214-20. [PubMed: 19661080]

44. Fillenbaum GG, Burchett BM, Blazer DG. Identifying a national death index match. Am J Epidemiol. 2009; 170:515-8. [PubMed: 19567777]

45. Sathiakumar N, Delzell E, Abdalla O. Using the National Death Index to obtain underlying cause of death codes. J Occup and Environ Med. 1998; 40:808-13. [PubMed: 9777565]

46. Huang L, Johnson KA, Mariotto AB, Dignam JJ, Feuer E. Population-based survival-curve analysis of ER-negative breast cancer. Breast Cancer Res and Treat. 2010 Epub 2010 Feb 4.

47. Foulkes WD, Reis-Filho JS, Narod SA. Tumor size and survival in breast cancer - a reappraisal. Nat Rev Clin Oncol. 2010 Epub 2010 Mar 23.

Clin Cancer Res. Author manuscript; available in PMC 2011 December 15. 

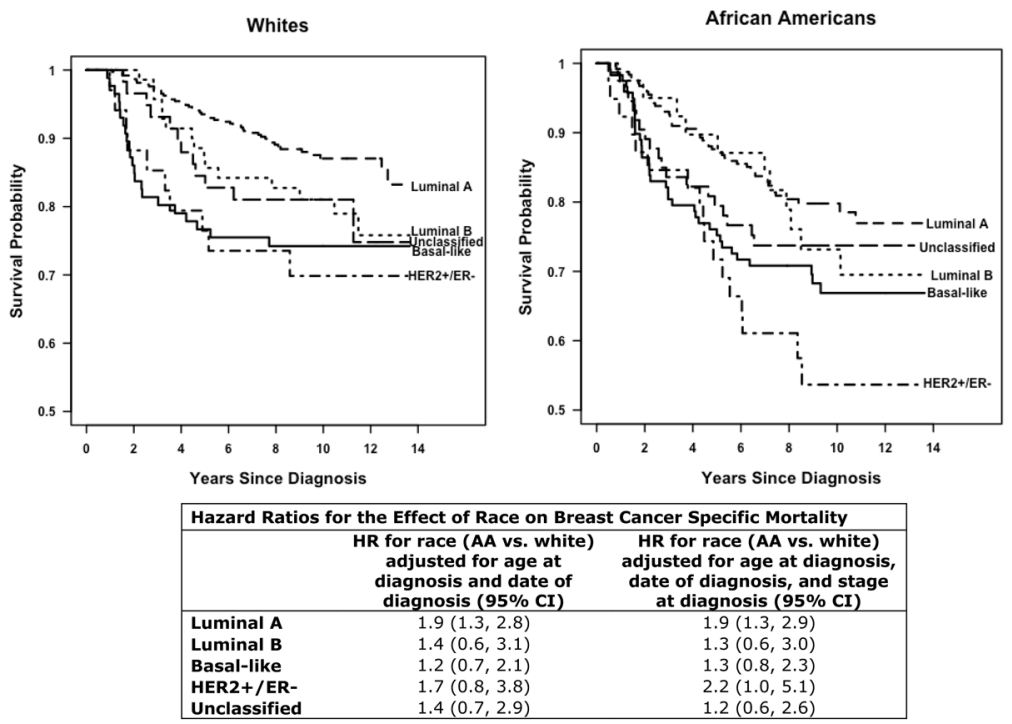

Figure 1.

Race-stratified Kaplan-Meier Plots and Race Effect Estimates for Breast Cancer Specific Mortality by Immunohistochemical Subtype in the Carolina Breast Cancer Study, 19932006 


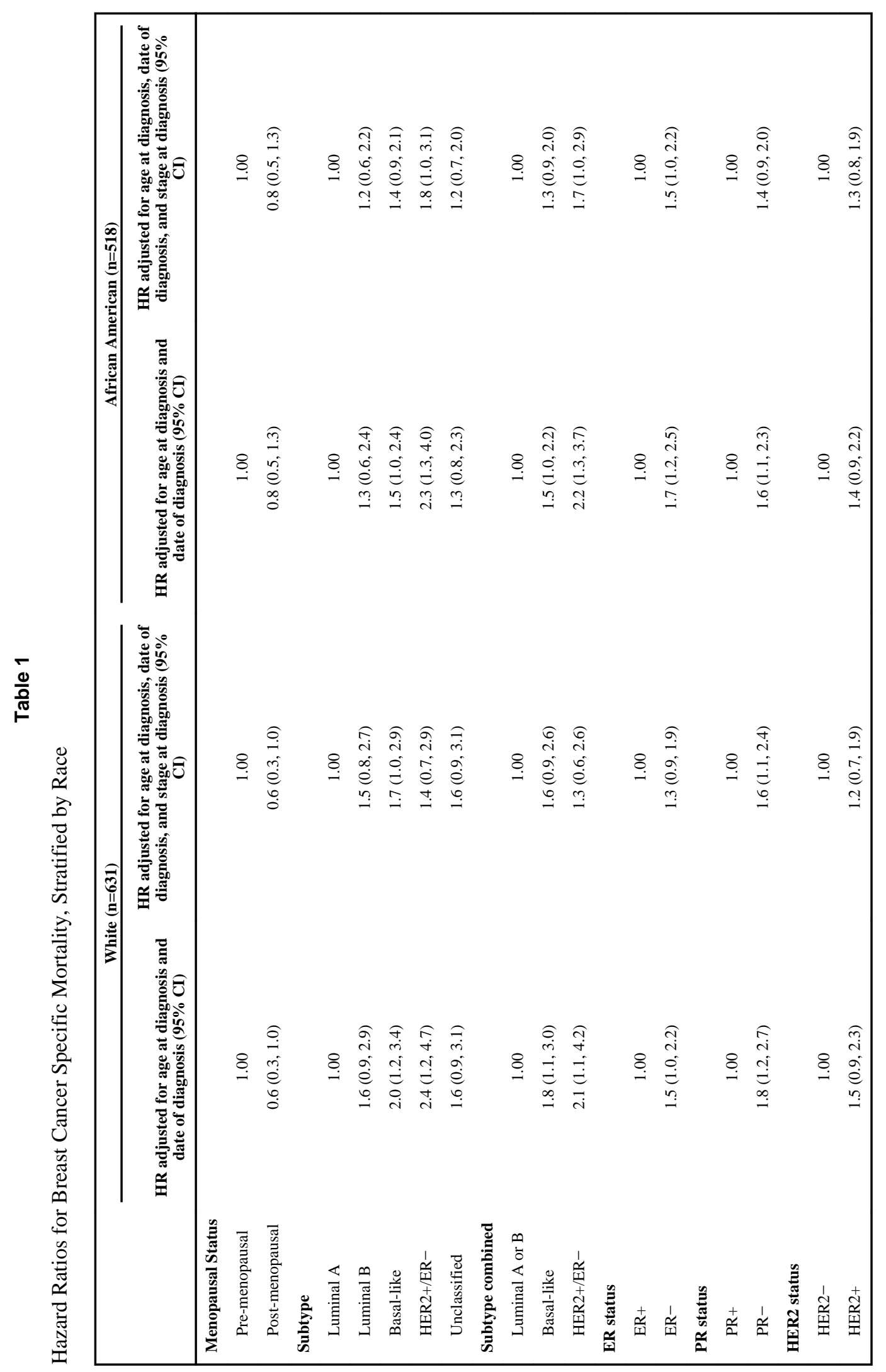

Clin Cancer Res. Author manuscript; available in PMC 2011 December 15. 
Table 2

Hazard Ratios for Breast Cancer Specific Mortality, Stratified by Race and Menopausal Status and Adjusted for Age at Diagnosis and Date of Diagnosis

\begin{tabular}{|c|c|c|c|c|}
\hline \multirow[b]{3}{*}{ Subtype } & \multicolumn{2}{|c|}{ White } & \multicolumn{2}{|c|}{ African American } \\
\hline & Premenopausal (n=323) & Postmenopausal $(\mathrm{n}=308)$ & Premenopausal (n=238) & Postmenopausal $(\mathrm{n}=\mathbf{2 8 0})$ \\
\hline & & & & \\
\hline Luminal A & 1.00 & 1.00 & 1.00 & 1.00 \\
\hline Luminal B & $1.2(0.6,2.5)$ & $2.9(1.0,8.4)$ & $1.2(0.5,2.9)$ & $1.3(0.5,3.6)$ \\
\hline Basal-like & $1.8(1.0,3.4)$ & $3.9(1.5,10.0)$ & $1.3(0.8,2.3)$ & $1.9(0.9,3.8)$ \\
\hline HER2+/ER- & $2.1(0.9,5.0)$ & $4.3(1.4,13.6)$ & $1.9(0.9,4.2)$ & $3.1(1.3,7.2)$ \\
\hline Unclassified & $1.4(0.7,3.0)$ & $2.1(0.6,7.4)$ & $1.0(0.5,2.0)$ & $1.9(0.8,4.3)$ \\
\hline \multicolumn{5}{|l|}{ Subtype combined } \\
\hline Luminal A or B & 1.00 & 1.00 & 1.00 & 1.00 \\
\hline Basal-like & $1.8(1.0,3.2)$ & $3.0(1.2,7.4)$ & $1.3(0.8,2.2)$ & $1.8(0.9,3.6)$ \\
\hline HER2+/ER- & $2.0(0.9,4.8)$ & $3.4(1.1,10.3)$ & $1.9(0.9,4.0)$ & $3.0(1.3,6.9)$ \\
\hline \multicolumn{5}{|l|}{ ER status } \\
\hline $\mathrm{ER}+$ & 1.00 & 1.00 & 1.00 & 1.00 \\
\hline ER- & $1.4(0.9,2.2)$ & $2.1(1.0,4.2)$ & $1.3(0.8,2.2)$ & $2.3(1.3,3.9)$ \\
\hline \multicolumn{5}{|l|}{ PR status } \\
\hline $\mathrm{PR}+$ & 1.00 & 1.00 & 1.00 & 1.00 \\
\hline PR- & $1.9(1.2,3.0)$ & $2.1(1.0,4.2)$ & $1.4(0.9,2.3)$ & $1.9(1.1,3.4)$ \\
\hline \multicolumn{5}{|l|}{ HER2 status } \\
\hline HER 2- & 1.00 & 1.00 & 1.00 & 1.00 \\
\hline HER $2+$ & $1.2(0.7,2.1)$ & $2.3(1.1,5.1)$ & $1.4(0.8,2.5)$ & $1.5(0.8,2.9)$ \\
\hline
\end{tabular}


Table 3

Time-Stratified Hazard Ratios (HRs), Breast Cancer Specific Mortality Adjusted for Race, Age at Diagnosis, and Date of Diagnosis

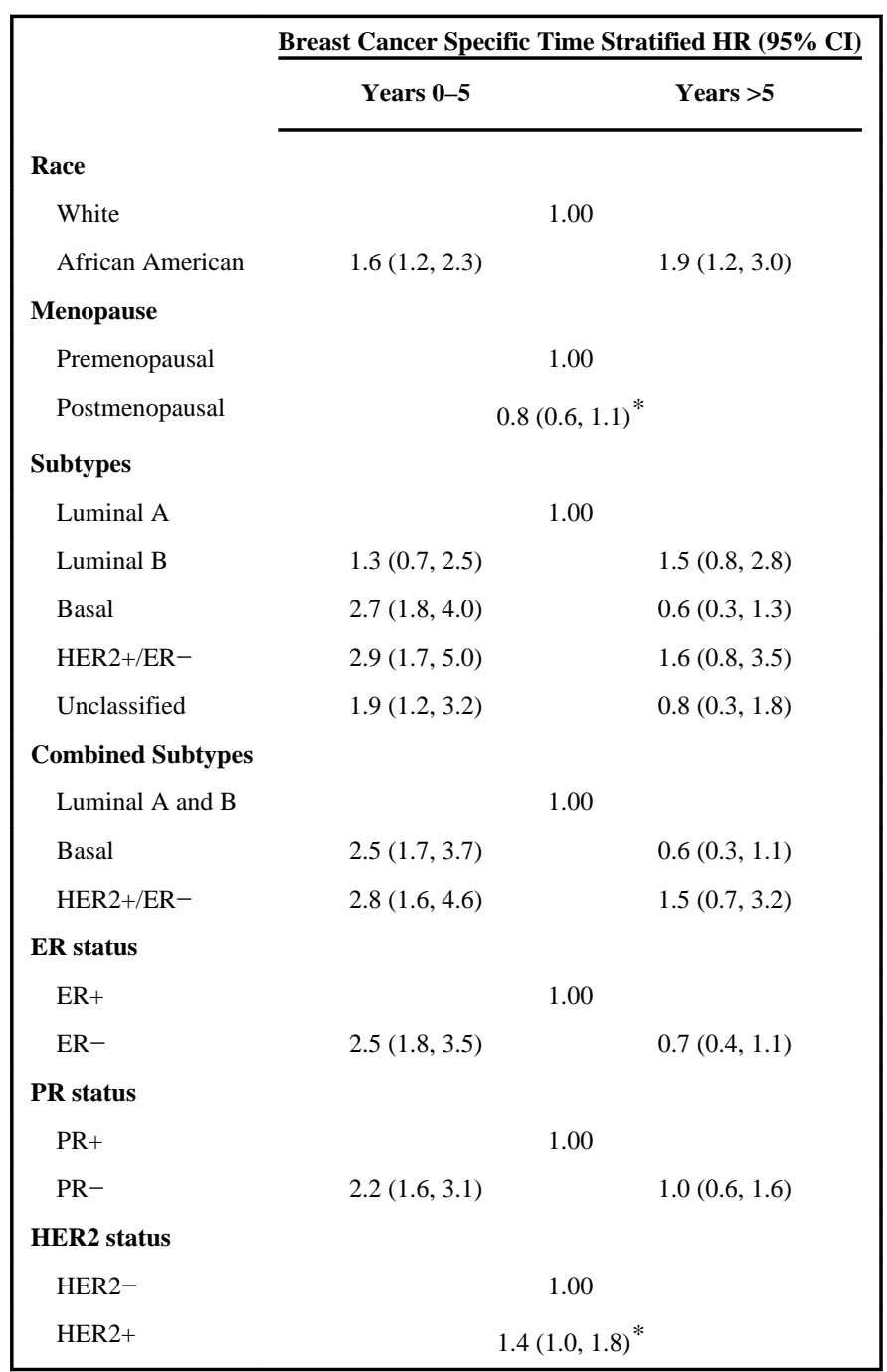

* single estimate given because proportional hazards assumption was not violated 


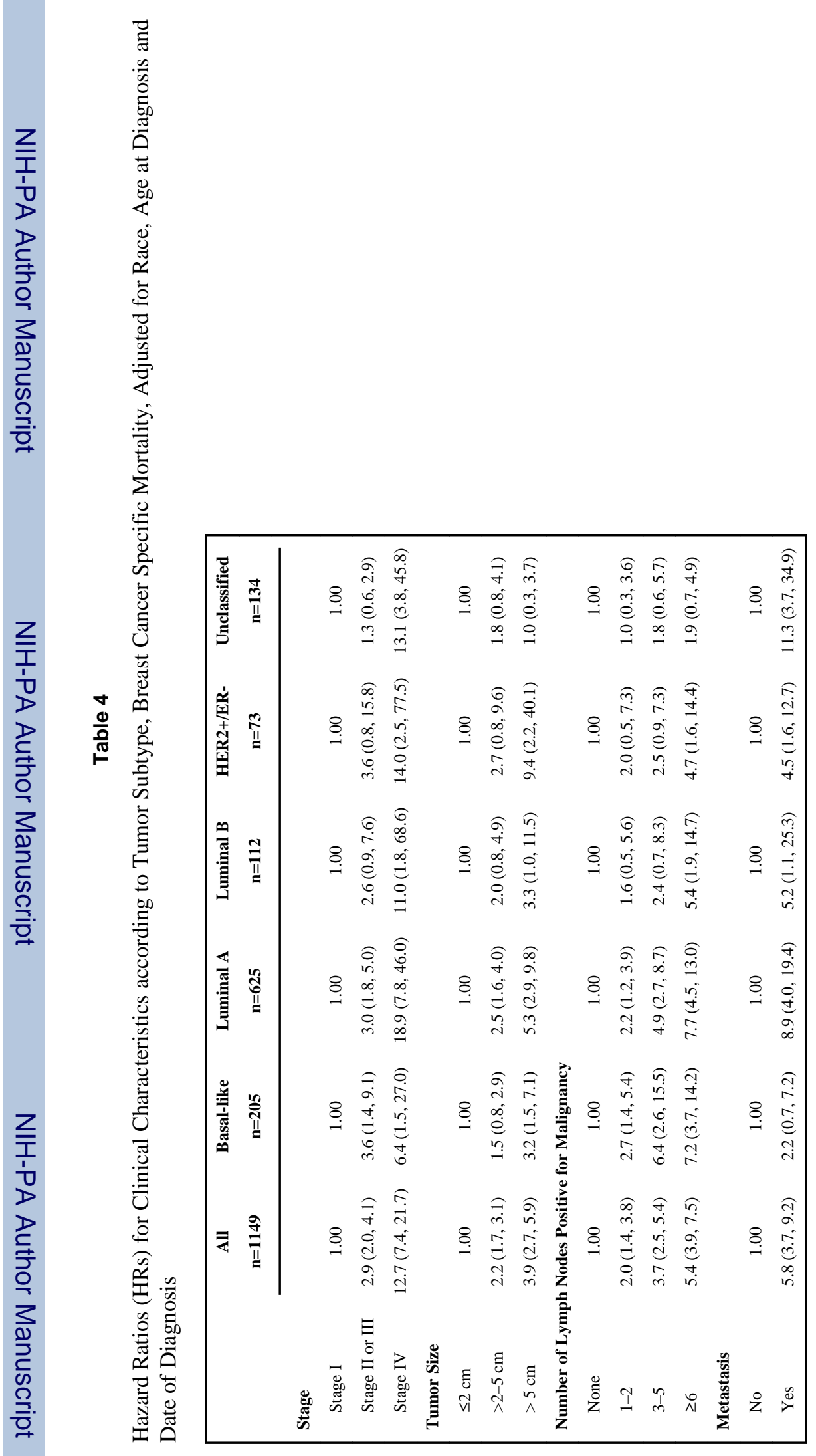

Clin Cancer Res. Author manuscript; available in PMC 2011 December 15. 


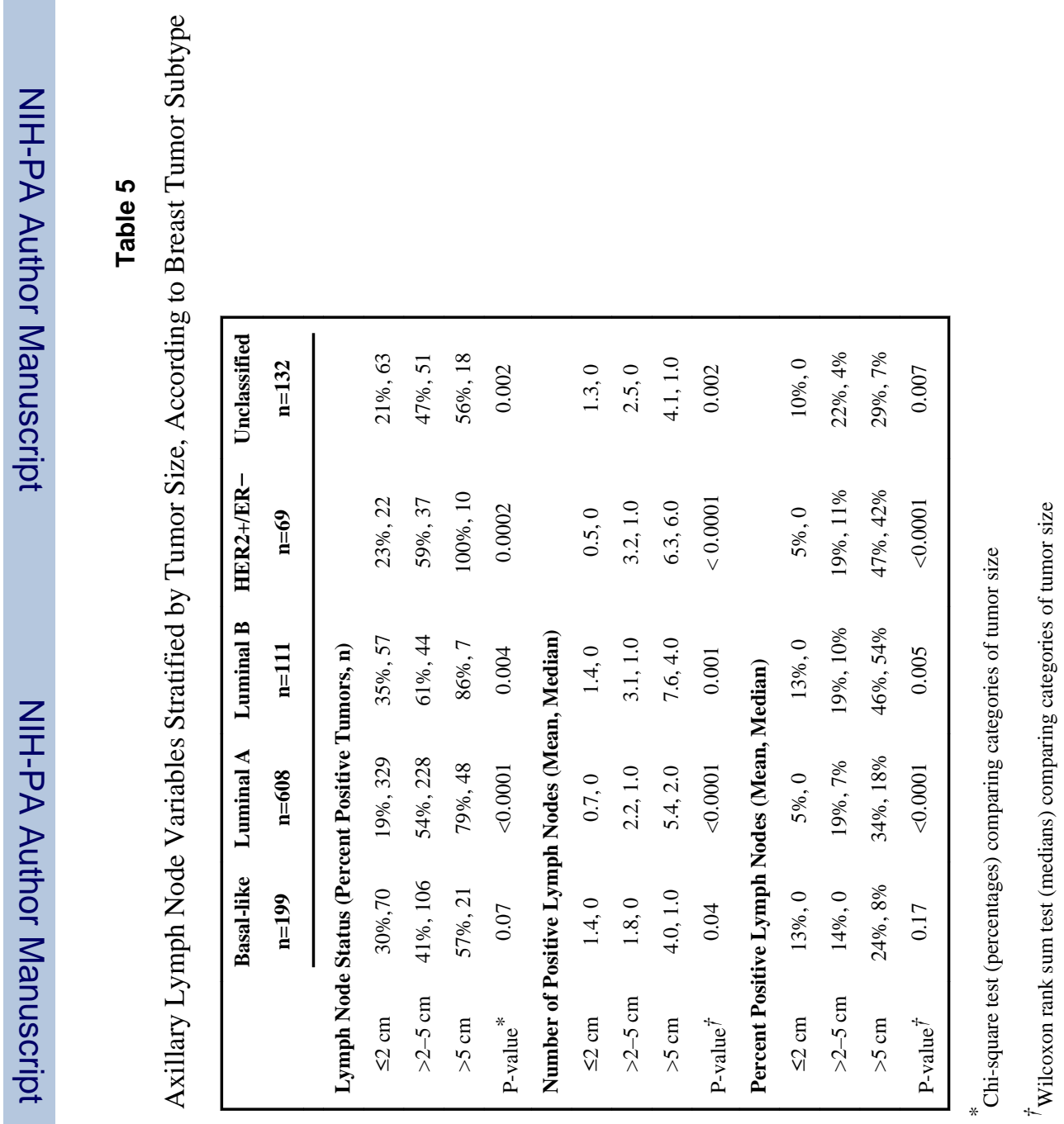

Clin Cancer Res. Author manuscript; available in PMC 2011 December 15. 